\title{
Next-Generation Allergen-Specific Immunotherapy for Japanese Cedar Pollinosis Using Molecular Approaches
}

\author{
Fumio Takaiwa ${ }^{1,2}$ \\ 'Soul Signal Institute, Kojyohama, Shiraoi, \\ Hokkaido, 059-064I, Japan; ${ }^{2}$ Institute of \\ Agrobiological Sciences, National \\ Agriculture and Food Research \\ Organization, Tsukuba, Ibaraki, 305-8602, \\ Japan
}

\begin{abstract}
Japanese cedar (JC) pollinosis is the most major IgE-mediated type I allergic disease in Japan. Allergen-specific immunotherapy is the only curative treatment for allergic diseases. Subcutaneous immunotherapy and sublingual immunotherapy have been introduced in Japan for JC pollinosis, but do not avoid some adverse side effects, because the natural allergens used as tolerogens cross-link with specific IgE types on mast cells and basophils. To make immunotherapy for JC pollinosis safer, more effective and convenient, rice-based oral allergy vaccines using hybrid peptides composed of multiple T-cell epitopes or recombinant deconstructed hypoallergenic derivatives derived from major allergens - Cry j 1 and Cry j 2 - have been developed and their efficacy and safety evaluated by oral administration of transgenic rice seeds. Furthermore, recombinant modified JC allergens conjugated with various immunomodulatory molecules and DNA-based vaccines have been created and their efficacy assessed.
\end{abstract}

Keywords: allergen-specific immunotherapy, T cell-epitope peptide, Japanese cedar pollinosis, rice-based oral vaccine, hypoallergenic derivatives

\section{Introduction}

Japanese cedar (JC; Cryptomeria japonica) pollinosis is a predominant seasonal allergic disorder and an important public-health problem in Japan. ${ }^{1,2}$ The prevalence of JC pollinosis has increased significantly since the first report in 1964, and affects $>30 \%$ of Japanese population. ${ }^{3-5}$ JC-pollen dispersal usually starts in early February, lasts for 10 weeks, and is followed by Japanese cypress pollen dispersal, which reaches its peak in late March. The major allergens causing JC pollinosis have been identified to be Cry J 1 and Cry J 2. It has been reported that $>90 \%$ of patients with JC pollinosis have IgE types specific to these allergens. Cry $\mathrm{j} 1$ is a basic glycoprotein homologous to pectate lyase with molecular weights of 41 and $45 \mathrm{kDa}$ and is localized in pollen-cell walls. ${ }^{6}$ Cry j 2 is a basic glycoprotein homologous to polymethyl galacturonate with a molecular weight of $45 \mathrm{kDa}$, which is located in pollen-starch granules. ${ }^{7,8}$ There is cross-reactivity between the pollen allergens of JC and Japanese cypress, because about $70 \%$ of patients with JC pollinosis are afflicted with Japanese cypress-pollen allergens. This is due to the fact that the Cry $\mathrm{j} 1$ and Cry j 2 allergens share $80 \%$ and $74 \%$ amino acid-sequence similarities with the homologues of Japanese cypress - Cha o 1 and Cha o 2 - thus suggesting the potential for cross-reactivity for some T-cell and B-cell epitopes.
Correspondence: Fumio Takaiwa Institute of Agrobiological Sciences, National Agriculture and Food Research Organization, Kannondai 3-I-3, Tsukuba, Ibaraki, 305-8604, Japan

Tel +8I-29-838-8373

Fax +83-29-838-8397

Email takaiwa@affrc.go.jp 
Cha o 3 has recently been characterized as a major cypress-pollen allergen in addition to Cha o 1 and Cha o 2. ${ }^{9}$ Cry j 4 is a homologue of Cha o 3 ( $85 \%$ identity) with cellulase function and a molecular weight of $61 \mathrm{kDa}$, but its presence is remarkably low in JC pollen compared to Cha o $3 .^{10}$ Several other minor JC-pollen allergens have been also identified. ${ }^{11}$ Cry j 3 is a thaumatin-like protein in the PR5 family with a molecular weight of $27 \mathrm{kDa}$ homologous to Jun a 3, a major allergen from mountain cedar pollen. CJP-4 from JC pollen has been identified as a $34 \mathrm{kDa}$ protein with endochitinase activity (class IV chitinase) that cross-reacts with latex allergens. CJP-6 has been identified as an isoflavone reductase-like allergen. The other JC-pollen allergens - CJP-8, CPA9, and CPA63 - have also been identified on allergenome analysis. These are homologous to lipid-transfer protein, subtilisin-like protease and aspartic protease, respectively.

The main treatments for pollinosis are avoidance of pollens and pharmacotherapy using antihistamines, antileukotrienes, and intranasal corticosteroids to block the release of chemical mediators from mast cells and basophils. These treatments relieve symptoms, but do not provide a cure for allergic diseases.

Allergen-specific immunotherapy (AIT) is the only clinically available curative treatment against IgEmediated type I allergic diseases, such as allergic rhinitis, conjunctivitis and asthma, and exhibits long-lasting effect even after discontinuation, ${ }^{12}$ ie, it can change the course of the disease. Immunotolerance to the causative allergens is induced by this treatment, which is conventionally achieved by subcutaneous injection with increasing doses of crude-pollen extract for 3-5 years. ${ }^{13,14}$ Used as a tolerogen, this crude extract is also sometimes accompanied by severe side effects, such as anaphylactic shock, which is caused by the cross-linking of allergen with a specific IgE capture of allergen on the surface of mast cells and circulating basophils. Furthermore, frequent medical visits for repeated painful injections are laborious, uncomfortable, and inconvenient. Subcutaneous immunotherapy (SCIT) for JC pollinosis has been administered in Japan since the 1960s.

Sublingual immunotherapy (SLIT) has been proposed as an alternative to SCIT. ${ }^{15}$ SLIT reduces the burden on patients because it can be administered at home and has fewer severe side effects. However, it takes longer to attain therapeutic efficiency than SCIT, and its effects are thought to be somewhat weaker.
Therefore, establishment of more convenient, more effective, and safer protocols is highly desired for a nextgeneration AIT. To overcome these problems, designing recombinant hypoallergenic derivatives retaining immunogenicity, but reducing allergenicity ( $\mathrm{IgE}$ activity), is an ideal strategy. Destruction of the conformational structure of allergens required by recognition of specific IgE types can be achieved by oligomerization, fragmentation, shuffling (reassembling of sequences), or deletion of disulfide bonds of the causative allergen sequence. ${ }^{16,17}$ Hybrid T cell-epitope peptides derived from allergens have been designed. ${ }^{18}$ Many clinical trials on allergy patients have been performed using these recombinant hypoallergenic derivatives, and their efficacy and safety have been evaluated in various allergies. Furthermore, conjugation of adjuvants with tolerogens and new routes of administration have been developed to allow improvement of efficacy and convenient administration.

\section{Administration Routes of Allergens for AIT Subcutaneous Immunotherapy}

SCIT typically requires a buildup phase of one to three injections per week for the first 3-6 months of treatment, with maintenance injections every $2-8$ weeks afterward for 3-5 years. Nonstandardized JC-pollen extract was launched in 1969 and used in clinical practice until 2000, when its standardization was achieved by the Japanese Allergology Society. SCIT with Japanese Society for Tuberculosis-standardized JC-pollen extracts has led to reduced clinical symptoms and medication scores, as well as improvement in quality of life (QOL). Reduction of JC pollen-specific IgE and basophil activation and elevation of $\mathrm{JC}$ pollen-specific $\mathrm{IgG}_{4}$ levels have been induced by SCIT over the long term (3-5 years).

\section{Sublingual Immunotherapy}

SLIT has been accepted as a viable alternative to SCIT for at least 30 years. SLIT can be administered at home, thus providing benefits in convenience and comfort. Theoretically, this aims to avoid the severe acute side reactions induced with large doses. SLIT with a new standardized JC pollen extract for JC pollinosis was approved in October 2014 by the Japanese Ministry of Health, Labour, and Welfare. ${ }^{21}$ At present, SLIT for JC pollinosis is achieved by placing the allergen under the tongue via either SLIT drops (Cedartolen) in an aqueous 
formulation of allergen extract or SLIT tablets (CedarCure) in a freeze-dried formulation. JC-pollen SLIT drops (Cedartolen) have usually been used at a dose of 2,000 JAU (Japanese allergy unit) containing 1.5-4.2 $\mu \mathrm{g}$ Cry j 1 as the maximum concentration for the maintenance phase. Although this dose has been reported to be effective in improvement of clinical symptoms for both children and adults, a higher dose is desired to enhance efficacy, because it has been shown to be doseand duration-dependent. ${ }^{22,23}$ To improve clinical efficacy and patient convenience, JC-pollen SLIT tablets 5,000 JAU/day was selected as the optimum dose and is used for treatment during the maintenance phase. ${ }^{23}$ However, the immunological effects of SLIT are lower than those of SLIT, since higher doses of the causative allergen (20- to 30 -fold) are required for SLIT to obtain the same therapeutic effect as SCIT. SLIT has a better safety profile than SCIT. Actually, the risk of systemic allergic reactions (severe anaphylactic shocks) is extremely decreased, whereas local side effects, such as oral itching and swelling, occur at a relatively high rate.

Large clinical SLIT trials for JC pollinosis have demonstrated significant reductions in total nasal symptoms and medical scores compared to placebo. ${ }^{24,25}$ The number of JC pollen-specific $\mathrm{T}_{\mathrm{h}} 2$ cytokine-producing cells increased during the pollen season in the placebo group, whereas increases were inhibited in the SLIT group. The number of JC pollen specific-induced Foxp $3^{+}$regulatory $\mathrm{T}$ cells $\left(\mathrm{T}_{\mathrm{reg}}\right)$ cells increased only in the SLIT group. SLIT induced tolerance to JC-pollen allergens by increasing specific serum $\mathrm{IgG}_{2}$ and $\mathrm{IgG}_{4}$ levels and allergen-specific $\mathrm{T}_{\text {reg }}$ cells producing IL10 and TGF $\beta .{ }^{26}$ When SLIT is carried out by treatment with JC-pollen SLIT drops for about 3 years, therapeutic effects, such as alleviation of clinical symptoms, reduction in pharmaceutical, use and improvement in QOL are maintained for at least 2 years, even after the cessation of treatment. ${ }^{27}$ Such symptomatic improvement is closely associated with the increase in serum allergen-specific $\mathrm{IgG}_{4}$ acting as blocking antibody to IgE.

\section{Oral Immunotherapy}

Oral immunotherapy is a promising method for modulating immunoresponse, since relatively large amounts of allergens can be orally administered and a large number of immune cells are present in gut-associated lymphoid tissue (GALT), providing suitable circumstances to induce immunotolerance to administered allergens.
The oral route is safe and convenient, and usually used as immunotherapy against food allergies. However, oral immunotherapy has been fundamentally abandoned for allergic rhinitis, because satisfactory clinical efficacy at both low and high does could not be obtained for several allergens (birch pollen and timothy-grass pollen allergen) in the $1980 \mathrm{~s}^{28}$ Side effects affecting the gastrointestinal tract were also reported in many patients in the activetreatment groups. These negative results might have been due to inadequate formulation of the allergens administered and the requirement for high amounts of allergens.

It has recently been recognized that cereal seed provides an effective delivery vehicle for antigens to GALT without severe degradation by digestive enzymes in the gastrointestinal tract when transgenic seed containing antigens (T cellepitope peptides or hypoallergenic derivatives of JC-pollen allergens) are orally administered. ${ }^{29}$ This is caused by the antigen deposited in protein bodies (PBs) of endosperm cells being bioencapsuled through double barriers of the cell wall and PBs exhibiting resistance to proteolytic enzymes and harsh conditions. ${ }^{30}$ It is known that recombinant proteins expressed in seed (endosperm) highly and stably accumulate in PBs devoid of proteases when produced as secretory proteins via the endoplasmic reticulum (ER). Furthermore, seed has many advantages as a production platform of recombinant proteins in terms of productivity, stability, safety, cost-effectiveness, and scalability. ${ }^{31}$

The amount or nature of the antigen determines the route of uptake into different parts of GALT such as the lamina propria or Peyer's patches for induction of oral tolerance. Particulate materials enter GALT mostly by M cell-mediated transcytosis, whereas soluble or small antigens are absorbed by epithelial cells and/or taken up by epithelium-associated macrophages, dendritic cells (DCs), or goblet cells. Oral tolerance is initiated by $\mathrm{CD}_{103}{ }^{+} \mathrm{DCs}$ that capture the antigen in the lamina propria. After contact with the antigen, $\mathrm{CD} 103^{+}$DCs migrate to the mesenteric lymph nodes and induce differentiation of naïve $\mathrm{T}$ cells into gut-forming regulatory Treg cells through a mechanism dependent on TGF $\beta$ and retinoic acid.

Regarding the underlying oral immunotolerance mechanism, $\mathrm{T}_{\text {reg }}$ cells play a critical role in developing and maintaining immunotolerance to allergens by producing inhibitory cytokines (IL10, TGF $\beta$, and IL35). ${ }^{32,33}$ Under normal conditions, it is known that repeated exposure at low doses of antigen promotes the development of $T_{\text {reg }}$ cells. In contrast, exposure to large amounts of antigen leads to T-cell anergy or Fas-mediated clonal deletion. 
$\mathrm{FOXP}^{+} \mathrm{iT}_{\text {reg }}$ cells are essential for establishing oral tolerance on exposure to inhaled allergens, since mutations in FOXP3 locus results in a loss of peripheral tolerance. Other $\mathrm{T}_{\text {reg }}$ cells, IL10-producing $\mathrm{T}_{\mathrm{r}} 1$ cells and TGF $\beta$-producing $\mathrm{T}_{\mathrm{h}} 3$ cells are also involved in peripheral oral tolerance. In particular, $\mathrm{T}_{\mathrm{h}} 3$ cells are characteristically induced after antigen feeding, and are implicated in the development of FOXP3 $^{+} \mathrm{iT}_{\text {reg }}$ cells via secreted TGF $\beta$. FOXP3 ${ }^{+} \mathrm{iT}_{\text {reg }}$ cells can condition DCs to induce IL10-producing $\mathrm{T}_{\mathrm{r}} 1$ cells by a mechanism dependent on IL27. $\mathrm{T}_{\mathrm{r}} 1$ cells suppress immunoreactions through IL10 production. IL10-producing $T_{r} 1$ and $B_{\text {reg }}$ cells contribute allergen tolerance through suppression of effector $T$ cells $\left(T_{h} 1\right.$ and $\left.T_{h} 2\right)$ and selective induction of blocking $\mathrm{IgG}_{4}$ antibodies via IL10 production.

\section{Other Immunotherapies Using Noninjection Routes}

Intralymphatic immunotherapy (ILIT) is a novel method for direct administration of allergens into lymph nodes. The major benefit of ILIT is the reduced dose of allergen and numbers of injection. Improved safety, efficacy, and convenience of ILIT have been also reported. This immunotherapy has recently been applied to JC-pollinosis patients. ${ }^{34} \mathrm{JC}$ pollen-allergen extracts were administered by three intralymphatic inguinal injections before the first pollen season. ILITtreated patients $(n=12)$ tended to show improved symptom and medication scores compared to placebo-treated ones $(n=6)$, though without statistical significance. When assessed by nasal provocation testing and visual analogue-scale scoring after the first season, the effectiveness of ILIT was significant. Notably, these effects continued until the second or third season. Neither allergen-specific antibodies nor $\mathrm{T}_{\text {reg }} /$ $\mathrm{B}_{\text {reg }}$ cells changed in peripheral blood.

Epicutaneous immunotherapy is another novel AIT method in which allergens are applied to the skin via patches containing a small dose of allergen. Administered allergens captured by Langerhans cells and epidermal DCs migrate to lymph nodes to prime $\mathrm{CD} 4^{+} \mathrm{T}$ cells. To date, this administration route has not yet been applied to AIT for JC pollinosis. This treatment has been put forward for immunotolerance induction against food allergies, such as peanut allergy. ${ }^{35}$

\section{Modification of Allergens (Design of Allergy Vaccines) \\ T Cell-Epitope Peptides}

T-cell epitopes were originally designed to modulate allergen-specific T-cell responses without IgE-mediated activation of effector cells. This was due to evidence that $\mathrm{T}$ cell-epitope peptides retained immunogenicity, but had little ability to cross-link the allergen-specific $\operatorname{IgE}(\operatorname{IgE}$ reactivity) on mast cells and basophils. As a result, T-cell epitopes can induce T-cell tolerance without side effects.

The underlying immunological mechanism of $\mathrm{T}$ cell epitope-based peptide immunotherapy is induction of specific anergy or deletion of allergen-specific $T_{h} 2$ cells, immunodeviation, and induction of $\mathrm{T}_{\text {reg }}$ cells, leading to IL10 production. $^{36}$ Allergen-specific protective $\mathrm{IgG}_{4}$ acting as a blocking antibody against $\mathrm{IgE}$ is not elicited, due to the short length of the peptide. Such an immunotolerance mechanism is associated with administration duration, tolerogen dose, and administration route. Repeated administration at low doses induces $\mathrm{T}_{\text {reg }}$ cells, which is called active suppression, while high-dose administration leads to T-cell anergy and deletion of effector $\mathrm{T}$ cells. $\mathrm{T}_{\text {reg }}$ cells are involved in inducing and maintaining specific unresponsiveness to allergens. Unresponsiveness is primarily maintained by IL10 and TGF $\beta$, which are produced by antigen-specific $\mathrm{T}_{\text {reg }}$ cells. Naturally occurring $\mathrm{FOXP}^{+}$and induced Treg cells (iTreg cells) contribute to the establishment of peripheral T-cell tolerance.

As for the efficacy of peptide oral immunotherapy for JC pollinosis, it was first reported that oral administration of Cry j 2 major T cell epitopes (p246-259) to model $\mathrm{BALB} / \mathrm{c}$ mice before challenge with the Cry j 2 allergen resulted in reduction of $\mathrm{T}$-cell proliferation and specific IgE and histamine release by mast cells. ${ }^{37}$ Alleviation of sneezing and airway obstruction were also observed. ${ }^{38}$ These results indicated that oral feeding of $\mathrm{T}$ cell-epitope peptides can induce immunotolerance in specific $\mathrm{T}$ cells. However, to obtain a similar effect to SCIT, $>100$-fold doses of tolerogen were required

To test a new immunotherapy based on the principle of seed-based oral tolerance, it was examined whether rice seed containing major T-cell epitopes derived from Cry $\mathrm{j} 1$ and Cry j 2 allergens could be utilized as a vehicle to deliver the tolerogen to GALT through oral administration. Major mouse T-cell epitopes from two allergens (p277-290 of Cry j 1 and p246-259 of Cry j 2) were expressed as part of the soybean storage protein glycinin $\left(\mathrm{A}_{1 \mathrm{a}} \mathrm{B}_{1 \mathrm{~b}}\right)$ under the control of the endosperm-specific rice glutelin GluB1 promoter in transgenic rice seed. ${ }^{39}$ When transgenic rice seeds were fed to BALB/c mice for 4 weeks prior to systemic challenges with JC pollen, not only significant reductions in specific $\mathrm{T}$ cell-proliferative reactions but also 
suppression of allergen-specific $\operatorname{IgE}$ and $\operatorname{IgG}$ levels and production of $\mathrm{T}_{\mathrm{h}} 2$ cytokines, IL4, IL5, and IL13 were induced compared to those of mice fed with nontransgenic rice seeds. Release of histamine from mast cells and sneezing regularity were restricted by oral administration of transgenic rice seeds. ${ }^{39}$ These results indicated that oral immunotolerance was definitely induced by oral administration of transgenic rice seeds containing T-cell epitopes.

For application of peptide immunotherapy to patients with JC pollinosis, multiple major T-cell epitopes were selected to overcome the variation of T-cell epitopes on the basis of different human leukemia-antigen (HLA) class II restrictions. Artificial hybrid peptides consisting of five to seven major human T cell epitopes with length of 13-25 amino acids derived from Cry j 1 and Cry j 2 were synthesized by recombinant technology. ${ }^{40-42}$ These hybrid peptides were designed to prevent IgE-mediated adverse effects. When the safety and efficacy of these hybrid peptides were evaluated, it was found that they did not bind to specific IgE antibodies in sera from patients with JC pollinosis, indicating no allergenicity of these hybrid peptides.40 Furthermore, they had $\mathrm{T}$ cell-proliferative activity comparable to the native Cry j 1 and Cry j 2 allergens. It is notable that the hybrid peptide had higher $\mathrm{T}$ cell-proliferation activity than the mixture of T-cell epitopes. ${ }^{40}$

The therapeutic efficacy of a hybrid peptide composed of six major human T-cell epitopes from Cry $\mathrm{j} 1$ and Cry $\mathrm{j}$ 2 (Cry-consensus peptide) was examined by subcutaneous administration once a week for 5 weeks to B10.S model mice sensitized with Cry j 1 and alum. ${ }^{42}$ In sensitized B10. $\mathrm{S}$ mice, a major T cell-epitope (peptides (p)211-225) of Cry $\mathrm{j} 1$ coincided with one of the prevalent epitopes of the Cry-consensus peptide. The hybrid peptide inhibited the production of Cry $\mathrm{j}$ 1-specific IgE, IL4, and IL5 $\left(\mathrm{T}_{\mathrm{h}} 2\right.$ mediated) and enhanced Cry j 1-specific $\operatorname{IgG}_{2 \mathrm{a}}$ and IFN $\gamma$ levels (shift to $\mathrm{T}_{\mathrm{h}} 1$ immunoresponse). Cry $\mathrm{j} 1$-induced sneezing and eosinophil infiltration in nasal tissues were also depressed. These results indicated that this hybrid peptide can induce immunotolerance to specific $\mathrm{T}$ cells.

Then, a hybrid peptide consisting of seven major human T-cell epitopes (7Crp) from Cry j 1 and Cry j 2 was produced in transgenic rice seeds and used as an oral vaccine. $^{43}$ To enhance accumulation in the edible part (endosperm) of rice seed, the codon-optimized 7Crp synthetic gene was expressed as a secretory protein by ligating the signal peptide and KDEL ER retention signal at its $\mathrm{N}$ and $\mathrm{C}$ termini under the control of the glutelin endosperm-specific promoter. ${ }^{43}$ The 7 Crp peptide was mainly deposited in ER-derived PBs in rice endosperm, whereas it did not accumulate in other tissue, such as leaf and stem, when expressed under the control of the ubiquitin constitutive promoter. $^{44}$

The efficacy of transgenic rice containing 7Crp was evaluated by oral administration to model mice and then subjected to clinical study in human subjects. When transgenic seeds were orally administered to B10.S mice with one common major T-cell epitope (p211-225 of Cry j 1), and then nasally challenged with Cry j 1 allergen, Cry j 1 specific $\mathrm{T}$ cell-proliferative response and specific- $\mathrm{IgE}$ levels were decreased compared with control mice fed nontransgenic rice seeds. ${ }^{43}$ This suggests that the $\mathrm{T}$ cellepitope in the hybrid peptide has the potential to suppress allergen-specific immunoreaction through the induction of immunotolerance to specific $\mathrm{T}$ cells (linked epitope suppression).

When the first clinical study of this transgenic rice in JC-pollinosis patients had been completed, it was found that allergen-specific T-cell proliferations against Cry j 1 and Cry j 2 allergens were significantly downregulated within just 2 weeks. ${ }^{45}$ This suppressive effect was dosedependent. On the other hand, when $80 \mathrm{~g}$ transgenic rice was orally administered for 20 weeks from December to the end of March, IL10 upregulation and IL13 downregulation were not associated with decreased cedar pollenspecific IgE antibodies or increased $\mathrm{IgG}_{4}$-blocking antibodies. There was no effect on IFN $\gamma$ and IL5 secretions from $\mathrm{T}_{\mathrm{h}} 1$ and $\mathrm{T}_{\mathrm{h}} 2$ cells, and the effect on IL4 was also minimal. This treatment did not accomplish improvement medication and QOL symptom scores. Significant improvements in symptom scores was restricted to the nose and eyes itching. As such, even though $\mathrm{T}$ cell-proliferative activity to Cry j 1 and Cry j 2 were highly suppressed, 80 g transgenic rice for 20 weeks did not give rise to a profound clinical improvement.

To examine the clinical efficacy of low-dose intake of transgenic rice for longer duration, two doses of transgenic rice seeds (5 $\mathrm{g}$ or $20 \mathrm{~g}$ ) were orally administered to patients for 24 weeks in each of two successive JC-pollen seasons. ${ }^{46}$ Specific T-cell proliferation following stimulation with 7Crp, Cry j 1, and Cry j 2 was significantly suppressed in the second JC-pollen season. No significant differences were detected among the three groups (5 g, 20 $\mathrm{g}$, and placebo) in the first season on clinical symptoms or medication scores. However, medication and face-scale scores improved in the $5 \mathrm{~g}$ transgenic rice group in the second season. 
These partial immunotolerance effects may be due to the possibility that 7Crp does not cover the full repertoire of $\mathrm{T}$ cell epitopes localized in Cry j 1 and Cry j 2 molecules due to the diversity of MHC (HLA) class II alleles in patients. Therefore, inclusion of additional T-cell epitopes to address MHC diversity may be necessary to accomplish significant clinical improvement. At present, transgenic rice expressing hybrid peptides composed of 12-13 T-cell epitopes covering most human T-cell epitopes has been generated. ${ }^{47}$ This transgenic rice may be expected to be broadly applicable as a next-generation oral peptide vaccine.

\section{Recombinant Hypoallergenic Derivatives}

Many B-cell epitopes are conformational, such that $\operatorname{IgE}$ reactivity is dependent on their correctly folded tertiary structure. As such, to reduce the allergenicity of Cry $\mathrm{j} 1$

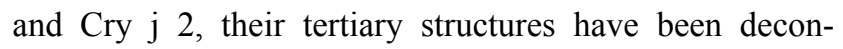
structed by fragmentation, shuffling (reassembly), and mutagenesis of Cys residues implicated in disulfide bonds. Transgenic rice expressing all molecules of deconstructed Cry j 1 and Cry j 2 was generated to treat a broader range of patients with different genetic backgrounds. ${ }^{48,49}$ These modified allergens preserve most T-cell epitopes, but lack B-cell epitopes. The reduction of allergenic and inflammatory activity from these allergens has been evaluated by IgE-binding assays, basophil-degradation tests, and basophil-activation tests. ${ }^{48-50}$

Tolerogenic efficacy of transgenic rice seed expressing the deconstructed Cry j 1 and Cry j 2 allergens has been evaluated in a murine model of allergic rhinitis. ${ }^{48}$ When mice fed transgenic seeds daily for 3 weeks were then challenged with $\mathrm{JC}$ pollen, allergen-specific $\mathrm{CD}^{+}{ }^{+} \mathrm{T}$-cell proliferation and specific $\operatorname{IgE}$ and $\operatorname{IgG}$ levels were significantly suppressed compared with mice fed nontransgenic rice seeds. It is notable that induction of blocking $\mathrm{IgG}_{4}$ or IL10 secreted from $T_{\text {reg }}$ cells was not detected, although production levels of the $T_{h} 2$ cytokines IL4, IL5, and IL13 were significantly depressed. Clinical symptoms of pollinosis, sneezing frequency, and infiltration of inflammatory cells (eosinophils and neutrophils) in nasal tissue were alleviated compared to controls. These results indicated that oral administration of transgenic rice seeds can induce immunotolerance to JC-pollen allergens, thus providing a promising approach using AIT for JC pollinosis.

Such prophylactic effects after transgenic rice intake have been also ascertained in an allergic conjunctivitis murine model. ${ }^{51}$ When mice were fed once a day for 20 days with transgenic rice seeds and then sensitized with two intraperitoneal injections of JC pollen, numbers of eosinophils and total inflammatory cells in the conjunctiva were significantly reduced in mice fed the transgenic rice seeds. Serum concentrations of both total and allergenspecific IgE were also significantly suppressed in mice fed the transgenic rice seeds. Furthermore, oral vaccination with transgenic rice resulted in significant downregulation of allergen-induced production of IL2, IL4, IL5, IL12p70, IFN $\gamma$, and IL17A by splenocytes, indicating the suppression of effector $T_{h} 1, T_{h} 2$, and $T_{h} 17$ cells. These results indicate that pollen-induced experimental allergic conjunctivitis in mice can be suppressed by oral administration of transgenic rice seeds.

Not only prophylactic but also curative effects have been observed after oral administration of this transgenic rice. When model mice were sensitized with $\mathrm{JC}$ pollen and then fed for 16 days with transgenic rice seeds, the number of eosinophils in the conjunctiva and clinical scores for conjunctivitis were significantly reduced compared to mice fed nontransgenic rice seeds. ${ }^{52}$ Although $\mathrm{T}_{\mathrm{h}}$ 2-cytokine IL4 and IL5 levels were not affected, IFN $\gamma$ was significantly enhanced. On the other hand, there was no significant difference in the number of $\mathrm{CD}^{+} \mathrm{CD} 25^{+} \mathrm{FOXP}^{+} \mathrm{T}_{\text {reg }}$ cells in the spleen or lymph nodes between mice fed transgenic and non-transgenic rice. These results suggested that increased IFN $\gamma$ production might contribute to suppression of conjunctival eosinophil infiltration in mice fed the transgenic rice.

Oral administration of transgenic rice containing deconstructed Cry j 1 and Cry j 2 demonstrated that blocking $\mathrm{IgG}_{4}$ or IL10 was not induced. This is different from the immunotolerance mechanism via $\mathrm{IgG}_{4}$ and $\mathrm{T}_{\text {reg }}$ cells observed in SCIT or SLIT using crude-pollen extract. Results from OIT using rice seeds suggested that immunotolerance may be induced through anergy or deletion of antigen-specific $\mathrm{T}$ cells. This difference may to be related to the antigen dose, antigen formulation, administration frequency, or administration route.

Next, before clinical studies in humans, transgenic rice seeds were orally administered to naturally sensitized Japanese monkeys exhibiting similar clinical allergic symptoms. ${ }^{53}$ Curative efficiency was suggested from results showing that suppression of allergen-specific PMBC proliferation was observed within just 2 months and allergen-specific IgE levels decreased in some monkeys. Furthermore, oral administration of transgenic rice to Japanese monkeys with JC pollinosis or healthy monkeys did not induce side effects. These findings indicate that 
transgenic rice containing hypoallergenic Cry $\mathrm{j} 1$ and Cry $\mathrm{j}$ 2 serves as a safe tolerogen.

\section{DNA Vaccines for AIT}

Nucleic acid-based vaccines have been shown to induce an immunological bias that prevents $T_{h} 2$ sensitization and drive immunoresponse toward $\mathrm{T}_{\mathrm{h}} 1$ via induction of IFN $\gamma$ and $\mathrm{IgG}_{2 \mathrm{a}}$ and suppression of $\mathrm{IgG}_{1}, \mathrm{IgE}$, and allergic inflammation. Furthermore, it is expected that nucleic acid-based vaccines have less risk of inducing severe side effects, such as anaphylaxis.

Repeated intramuscular inoculation of $\mathrm{BALB} / \mathrm{c}$ mice with pCACJ1 (plasmid DNA encoding Cry j 1) elicited a predominant $\mathrm{T}_{\mathrm{h}}$ 1-type immunoresponse $\left(\mathrm{IgG}_{2 \mathrm{a}}\right.$ production) and suppressed $\operatorname{IgE}$ and $\mathrm{IgG}_{1}$ responses. ${ }^{54}$ Splenic $\mathrm{T}$ cells isolated from pCACJ1-inoculated mice secreted IFN $\gamma$ but not IL4 upon stimulation with Cry $\mathrm{j} 1$, as well as its major T-cell epitope (p277-288). These findings suggest that intramuscular inoculation with pCACJ1 effectively elicits Cry j 1-specific $\mathrm{T}_{\mathrm{h}} 1$-type immunoresponses, resulting in inhibition of the IgE response to Cry $\mathrm{j} 1$.

To establish a new immunotherapy for type I allergic diseases without allergic side effects, MHC class II targeting DNA vaccine was developed using an invariant chain (Ii). This DNA vaccine for JC pollinosis was composed of the Cry j 2 major T-cell epitope and Ii for the delivery of the epitope peptide into the MHC class II-loading pathway. The Cry j 2 major T-cell epitope (p247-258) was inserted into the li by replacement with the core of class IIassociated invariant chain peptide (CLIP) or by fusion to the $\mathrm{C}$ terminus of the li, called pCPCJ2 and pliCJ2, respectively. ${ }^{55}$ When BALB/c mice were repeatedly inoculated with these plasmid DNAs intramuscularly, a $\mathrm{T}_{\mathrm{h}} 1$-cell response specific to p247-258 was induced without eliciting IgG-antibody production, resulting in inhibition of the subsequent $\operatorname{IgE}$ response to Cry j 2 immunization.

Lysosomal-associated membrane protein-1 (LAMP-1) is known to be a resident protein of lysosomes. Its lysosometargeting property has been used in the DNA-vaccine field. It has been shown that inclusion of LAMP significantly enhances both cellular and humoral responses in vaccinated animals. When DNA vaccines encoding CryJ1-LAMP and CryJ2-LAMP fusions were administered to BALB/c mice, high levels of IFN $\gamma$ and anti-Cry j 1/anti-Cry j 2-specific $\mathrm{IgG}_{2 \mathrm{a}}$ and low levels of $\mathrm{IgE}$ were induced, suggesting that $\mathrm{T}_{\mathrm{h}} 1$ response was predominantly elicited. ${ }^{56}$ After repeated allergen exposure, vaccinated mice were well protected, as indicated by a minimal level of allergen-specific IgE. These results suggest that the CryJ-LAMP vaccine has potential as effective therapy for JC pollinosis by skewing the allergy reaction from $T_{h} 2$ toward a $T_{h} 1$-dominant response. Robust and long-lasting $\mathrm{T}_{\mathrm{h}} 1$-type immunoresponse is expected to be triggered by LAMP-based DNA vaccination.

Safety and immunological effects of the CryJ2-LAMP DNA vaccine were evaluated in phase IA and IB clinical trials. ${ }^{57}$ Both $\mathrm{JC}$ and/or mountain cedar atopic human subjects were vaccinated with CryJ2-LAMP plasmid 4 times in the Phase IA trial and some subjects were boosted once in the Phase IB stage. The safety and immunologic biomarkers were assessed by adverse events, skin prick test for allergens and antibody detection in these subjects for an accumulated time from Day 0 of the Phase IA to the end of the Phase IB. The results indicated that CryJ2LAMP DNA vaccine is safe and may be immunologically effective in treating JC-induced allergy.

\section{Allergens Coupled with Immunomodulatory Compounds Galactomannan-Coupled Allergens}

When orally administered, conjugation of Cry j 1 with galactomannan has been shown to protect against Cry $\mathrm{j} 1$ protein enzymatically digesting in the stomach. No severe adverse events have been observed in subjects receiving Cry j 1-galactomannan. ${ }^{58}$ This is due to the fact that IgErecognition sites in Cry j 1 are masked by the conjugated galactomannan. Furthermore, galactomannan is known to accelerate the uptake of Cry $\mathrm{j} 1$ antigen into gut DCs. When orally administered to patients with JC pollinosis for 4 months from January to March, allergen-specific $\mathrm{IgG}_{4}$ and IL10 production were enhanced in PBMCs when compared to the control group. Furthermore, improvement in QOL scores and reductions in total symptom scores, medication scores, and total symptom-medication scores were observed throughout the pollen season. ${ }^{59}$ These results indicated that oral administration of Cry j 1galactomannan conjugates provides rapid, effective, and convenient immunotherapy. Galactomannan acts as a carrier and adjuvant for induction of tolerance to JC-pollen allergen.

\section{CpG-Conjugated Allergens}

Conjugating immunostimulatory sequences, such as $\mathrm{CpG}$ motifs, with allergens has been reported to enhance the effect of DNA for AIT. These sequences bind to toll like receptor 9 (TLR9), which is expressed within endosomes 
of human plasmacytoid DCs and B cells and thus promotes $T_{h} 1$ responses. Therefore, the allergenic activities of allergens are expected to be reduced by coupling of $\mathrm{CpG}-$ motif sequences with allergens.

When Cry j 1 was conjugated with $\mathrm{CpG}$ oligodeoxynucleotide and tested in a mouse model, strong allergenspecific $\mathrm{T}_{\mathrm{h}} 1$ responses, such as Cry $\mathrm{j} 1$-specific $\operatorname{IgG}_{2 \mathrm{a}}$ response and production of IL12, were elicited in their splenocytes. ${ }^{60}$ Moreover, higher IFN $\gamma$ levels were produced by $\mathrm{CD}^{+} \mathrm{T}$ cells of spleens in mice stimulated with $\mathrm{CpG}$ conjugated with Cry j 1 . These results indicated that Cry j 1-CpG immunization can induce Cry j 1-specific $\mathrm{T}_{\mathrm{h}} 1$ immunoresponses. Furthermore, $\mathrm{CpG}$ conjugation reduced binding to $\mathrm{IgE}$ in JC-pollinosis patients, indicating $\operatorname{IgE}$ epitopes on Cry $\mathrm{j} 1$ were masked by $\mathrm{CpG}$. As a result, Cry j 1-specific IgE levels were suppressed by CpG-Cry j 1 treatment.

Furthermore, T-cell epitopes derived from Cry j 2 was conjugated with $\mathrm{CpG}$ oligodeoxynucleotide and tested in mice. T-cell epitope-CpG treatment suppressed Cry j 2specific IgE reactions and attenuated clinical symptoms upon allergen challenge. ${ }^{61} \mathrm{~T}_{\mathrm{h}}$ 2-cytokine response (IL4 and IL5 production) was also significantly suppressed.

\section{Oligomannose-Coated Liposomes Entrapped with Allergens}

Antiallergenic effects following treatment with Cry $\mathrm{j} 1$ entrapped with oligomannose-coated liposomes (Cry j 1OMLs) were investigated in BALB/c mice sensitized with Cry j $1 .{ }^{62}$ When unsensitized mice were pretreated with Cry $\mathrm{j}$ 1-OMLs and then sensitized with Cry $\mathrm{j} 1$, total and allergenspecific IgE levels were suppressed. Injection of Cry j 1-OMLs induced robust $\mathrm{T}_{\mathrm{h}} 1$-cytokine responses (IFN $\gamma$ production) and Cry j 1-specific $\operatorname{IgG}_{2 a}\left(T_{h} 1\right.$-type antibody) production. These results indicated that allergen-specific $T_{h} 1$ immuneoresponses were preferentially induced with OMLs.

\section{Polyethylene Glycol-Conjugated Allergens}

To destroy the Cry j 1 and Cry j 2 conformational structures, the allergens were fused. Furthermore, a majority of Cys residues were replaced with serine residue to delete disulfide bonds required for maintenance of tertiary structures. ${ }^{63}$ This modified Cry j 1-Cry j 2 fusion protein was synthesized in Escherichia coli and conjugated with polyethylene glycol (PEG). When the purified PEGylated Cry j 1-Cry j 2 was subcutaneously injected into Cry $\mathrm{j}$ 1-sensitized mice and JC pollen-sensitized monkeys and then subcutaneously or intranasally challenged with Cry j 1 or JC pollen, Cry j 1-specific IgE levels were significantly suppressed, while CryJ1-specific IgG levels were increased in the PEGfusion-treated group. When splenocytes were stimulated with Cry $\mathrm{j} 1$, Cry j 1-specific T-cell proliferation and $\mathrm{T}_{\mathrm{h}} 2$ cytokine (IL5, IL13) production were significantly reduced, whereas $\mathrm{T}_{\mathrm{h}}$ 1-type IFN $\gamma$ was significantly increased. These results indicated PEG-Cry j 1-Cry j 2 elicited a $\mathrm{T}_{\mathrm{h}}$ 1-skewed immunoresponse. Therefore, PEG fusion is a suitable candidate as a safer and more effective vaccine for AIT.

\section{CTB-Fused Allergens}

Cholera toxin B (CTB) subunit has been used as an efficient mucosal carrier molecule for induction of oral tolerance. A mouse-type hybrid T cell-epitope peptide (3Crp) from Cry j 1 and Cry j 2 was expressed in rice seed as a fusion protein with either CTB or rice glutelin as a control. When these transgenic rice seeds were orally administered to $\mathrm{BALB} / \mathrm{c}$ mice and then challenged with JC pollen, rice seed containing $\mathrm{CTB} / 3 \mathrm{Crp}$ suppressed allergen-specific $\operatorname{IgE}$ responses and pollen-induced clinical symptoms (histamine release, sneezing frequency) at 50-fold lower doses than required when using control seed. ${ }^{64}$ These results indicated that the linked CTB had adjuvant activity for induction of oral immunotolerance.

\section{Conclusion}

AIT is the treatment of allergic disease by suppressing the immune system. The basic principle of AIT is to induce immunotolerance (a state of unresponsiveness of the immune system) to allergens through multiple cellular and molecular mechanisms by administering causative allergens. Immunotolerance is known to be regulated by such factors as T-cell anergy, apoptosis, $\mathrm{T}_{\text {reg }}$ cells, $\mathrm{B}_{\text {reg }}$ cells, suppressive cytokines, and DCs. SCIT and SLIT are used as effective treatment of various pollen-allergy diseases, including JC pollinosis, which leads to long-lasting effects even after cessation of treatment. SLIT has come to be the main one used because it is safer, painless, and comfortortable. However, there remain several drawbacks, such as risk of adverse reactions, long treatment duration ( $>3$ years), and low efficiency.

To overcome these problems observed in SCIT and SLIT, it is necessary to develop allergy vaccines with high efficiency (immunogenicity) without side effects 
(IgE reactivity). Furthermore, to improve immunoefficacy and patient convenience, administration routes and adjuvants leading to immunoenhancement also have to be taken into consideration (Figure 1).

Regarding efficacy and safety, allergenicity in $\operatorname{IgE}$ binding sites (B-cell epitopes) causing adverse reactions have been eliminated or reduced by destroying the tertiary structure of allergens while retaining the immunogenicity. Reduced allergenicity of destroyed Cry j 1 and Cry j 2 can be confirmed in vitro by IgE-binding and basophil-activation assays. Utilization of recombinant hypoallergenic derivatives or hybrid $\mathrm{T}$ cell-epitope peptides composed of multiple major T-cell epitopes derived from causative allergens can be expected to enhance efficacy and safety compared to native allergens. This can be explained by the fact that high-dose administration of allergen with reduced allergenicity (hypoallergenic derivative) allows reduced treatment duration, leading to patient convenience. However, late-phase side effects are still observed for immunotherapy using such hypoallergenic derivatives. This is due to late-phase allergic symptoms caused by Tcell activation based on preservation of T-cell epitopes.

To eliminate both IgE- and T cell-mediated side effects, a carrier-bound B cell epitope-based allergy vaccine has been developed and a clinical trial is already under way grasspollen allergy. ${ }^{65}$ This vaccine is produced by fusing hypoallergenic allergen peptides (B-cell epitopes of length 20-40 amino acids) derived from IgE-binding sites with an immunogenic viral carrier protein. This vaccine reduces IgE- and $\mathrm{T}$ cell-mediated side effects and induces robust allergen-specific $\mathrm{IgG}_{4}$-blocking antibodies. Such a B cell epitope-based allergy vaccine for JC pollinosis has not yet been developed. Conjugation of adjuvant with tolerogens improves efficacy and safety through a shift to $\mathrm{T}_{\mathrm{h}} 1$ or modulation of immunological properties, leading to reduction in administered doses and treatment duration. The administration route of tolerogen is associated with not only uptake by key immunoeffector cells but also patient convenience. Therefore, given that

\section{Administration route}

\section{Injection}

- Subcutaneous Immunotherapy

- Intralymphatic Immunotherapy

- Intramuscular injection of DNA

\section{Mucosal administration}

- Sublingual Immunotherapy

- Oral Immunotherapy

- Epicutaneous Immunotherapy

- Nasal Immunotherapy

\section{Modification of allergen \\ Chemical Modification \\ Hypoallergenic derivatives \\ - Destruction of tertiary structure \\ - Fragmentation \\ - Shuffling (reassembly) \\ - Disruption of disulfide bonds \\ T cell epitope peptide \\ Peptide carrier bound B cell epitope}

\section{Enhancing immunogenicity}

CpG conjugation (Th1 adjuvand)

Galactomannan conjugation

Oligomannose coated lyposome (Th1 adjuvand)

Polyethlen glycol conjugation (Th1 response)

CTB fusion

LAMP1 fusion

Monophosphoryl lipid A

\section{Future allergen-specific immunotherapy} using new allergy vaccines

- High efficiency

- Safety

- Convenience

- Manufactuability

Figure I Strategy for development of next-generation allergy vaccines. Next-generation allergen-specific immunotherapy for typel allergies, including JC pollinosis, that exhibits high efficiency, improved safety, and patient convenience, has to be developed through modification of allergens and use of suitable adjuvants. The administration route also has to be taken into account. 
hypoallergenic derivatives conjugated with suitable adjuvant molecule are administered using an appropriate route, immunotolerance to allergens can be effectively induced by much lower doses for shorter durations, resulting in safe, comfortable, and effective AIT for JC pollinosis.

\section{Disclosure}

The author reports no conflicts of interest in this work.

\section{References}

1. Yamada T, Saito H, Fujieda S. Present state of Japanese cedar pollinosis: the national affliction. $J$ Allergy Clin Immunol. 2014;133 (3):632-639. doi:10.1016/j.jaci.2013.11.002

2. Okamoto Y, Horiguchi S, Yamamoto H, et al. Present situation of cedar pollinosis in Japan and its immune responses. Allergol Int. 2009;58(2):155-162. doi:10.2332/allergolint.08-RAI-0074

3. Okubo K, Kurono Y, Ichimura K, et al. Japanese guidelines for allergic rhinitis 2020. Allergol Int. 2020;69(3):331-345. doi:10.1016/j.alit.2020.04.001

4. Sakashita M, Hirota T, Harada M, et al. Prevalence of allergic rhinitis and sensitization to common aeroallergens in a Japanese population. Int Arch Allergy Immunol. 2010;151(3):255-261. doi:10.1159/ 000242363

5. Urashima M, Asaka D, Endo T, et al. Japanese cedar pollinosis in Tokyo residents born after massive national afforestation policy. Allergy. 2018;73(12):2395-2397. doi:10.1111/all.13575

6. Sone T, Komiyama N, Shimizu K, et al. Cloning and sequencing of cDNA coding for Cry j I, a major allergen of Japanese cedar pollen. Biochem Biophys Res Commun. 1994;199(2):619-625. doi:10.1006/ bbrc.1994.1273

7. Komiyama N, Sone T, Shimizu K, et al. cDNA cloning and expression of Cry j II the second major allergen of Japanese cedar pollen. Biochem Biophys Res Commun. 1994;201(2):1021-1028. doi:10.1006/bbrc.1994.1804

8. Namba M, Kurose M, Torigoe K, et al. Molecular cloning of the second major allergen, Cry j II, from Japanese cedar pollen. FEBS Lett. 1994;353(2):124-128. doi:10.1016/0014-5793(94)01022-6

9. Osada T, Harada T, Asaka N, et al. Identification and gene cloning of a new major allergen Cha o 3 from Chamaecyparis obtusa (Japanese cypress) pollen. J Allergy Clin Immunol. 2016;138(3):911-913. doi:10.1016/j.jaci.2016.03.026

10. Osada T, Tanaka Y, Yamada A, et al. Identification of Cha o 3 homolog Cry j 4 from Cryptomeria japonica (Japanese cedar) pollen: limitation of the present Japanese cedar-specific ASIT. Allergol Int. 2018;67(4):467-474. doi:10.1016/j.alit.2018.02.004

11. Fujimura T, Kawamoto S. Spectrum of allergens for Japanese cedar pollinosis and impact of component-resolved diagnosis on allergenspecific immunotherapy. Allergol Int. 2015;64(4):312-320. doi:10.1016/j.alit.2015.05.008

12. Frew AJ. Allergen immunotherapy. $J$ Allergy Clin Immunol. 2010;125:S306-313. doi:10.1016/j.jaci.2009.10.064

13. Larche M, Akdis CA, Valenta R. Immunological mechanisms of allergen-specific immunotherapy. Nature Rev Imunol. 2006;6 (10):761-771. doi:10.1038/nri1934

14. Akdis CA. Therapies for allergic inflammation: refining strategies to induce tolerance. Nat Med. 2012;18(5):736-749. doi:10.1038/ nm. 2754

15. Radulovic S, Wilson D, Calderon M, Durham S. Systematic reviews of sublingual immunotherapy (SLIT). Allergy. 2011;66(6):740-752. doi:10.1111/j.1398-9995.2011.02583.x

16. Linhart B, Valenta R. Molecular design of allergy vaccines. Curr Opin Immunol. 2005;17(6):646-655. doi:10.1016/j.coi.2005.09.010
17. Valenta R, Ferreira F, Focke-Tajkl M, et al. From allergen genes to allergy vaccines. Ann Rev Immunol. 2010;28:211-241. doi:10.1146/ annurev-immunol-030409-101218

18. Larché R, Wraith DC. Peptide-based therapeutic vaccines for allergic and autoimmune diseases. Nat Med. 2005;11:S69-76. doi:10.1038/ nm1226

19. Inuo C, Ando H, Tanaka K, et al. Long-term immunological effects of Japanese cedar pollen-based subcutaneous immunotherapy. Allergol Int. 2018;67(3):408-410. doi:10.1016/j.alit.2017.11.002

20. Passalacqua G, Bagnasco D, Canonica GW. 30 years of sublingual immunotherapy. Allergy. 2020;75(5):1107-1120. doi:10.1111/ all.14113

21. Ohashi-Doi K, Lund K, Mitobe Y, Okamiya K. State of the art: development of a sublingual allergy immunotherapy tablet for allergic rhinitis in Japan. Biol Pharm Bull. 2020;43(1):41-48. doi:10.1248/bpb.b19-00093

22. Yonekura S, Gotoh M, Kaneko S, et al. Treatment duration-dependent efficacy of Japanese cedar pollen sublingual immunotherapy: evaluation of a Phase II/III trial over three pollen dispersal seasons. Allergol Int. 2019;68(4):494-505. doi:10.1016/j.alit.2019.05.002

23. Gotoh M, Yonekura S, Imai T, et al. Long-term efficacy and dosefinding trial of Japanese cedar pollen sublingual immunotherapy tablet. J Allergy Clin Immunol Pract. 2019;7(4):1287-1297. doi:10.1016/j.jaip.2018.11.044

24. Okamoto Y, Okubo K, Yonekura S, et al. Efficacy and safety of sublingual immunotherapy for two seasons in patients with Japanese cedar pollinosis. Int Arch Allergy Immunol. 2015;166 (3):177-188. doi:10.1159/000381059

25. Fujimura T, Yonekura S, Taniguchi $Y$, et al. The induced regulatory $T$ cell level, defined as the proportion of IL-10(+)Foxp3(+) cells among $\mathrm{CD} 25(+) \mathrm{CD} 4(+)$ leukocytes, is a potential therapeutic biomarker for sublingual immunotherapy: a preliminary report. Int Arch Allergy Immunol. 2010;153(4):378-387. doi:10.1159/000316349

26. Fujimura T, Yonekura $S$, Horiguchi $S$, et al. Increase of regulatory $T$ cells and the ratio of specific $\operatorname{IgE}$ to total $\operatorname{IgE}$ are candidates for response monitoring or prognostic biomarkers in two-year sublingual immunotherapy (SLIT) for Japanese cedar pollinosis. Clin Immunol. 2011;139(1):65-74. doi:10.1016/j.clim.2010.12.022

27. Nomura Y, Okubo K, Nakamura T, et al. Long-term treatment of Japanese cedar pollinosis with Japanese cedar pollen SLIT drops and persistence of treatment effect: a post-marketing clinical trial. Allergol Int. 2021;70(1):96-110. doi:10.1016/j.alit.2020.05.008

28. Canonica GW, Passalacqua G. Noninjection routes for immunotherapy. J Allergy Clin Immunol. 2003;111(3):437-448. doi:10.1067/ mai.2003.129

29. Takaiwa F. Update of the use of transgenic rice seeds in oral immunotherapy. Immunotherapy. 2013;5(3):301-312. doi:10.2217/imt.13.4

30. Takaiwa F, Wakasa Y, Takagi H, Hiroi T. Rice seed for delivery of vaccines to gut mucosal immune tissues. Plant Biotechnol J. 2015;13 (8):1041-1055. doi:10.1111/pbi.12423

31. Takaiwa F, Wakasa Y, Hayashi S, Kawakatsu T. An overview on the strategies to exploit rice endosperm as production platform for biopharmaceuticals. Plant Sci. 2017;263:201-209. doi:10.1016/j. plantsci.2017.07.016

32. Mayer L, Shao L. Therapeutic potential of oral tolerance. Nat Rev Immunol. 2004;4(6):407-419. doi:10.1038/nri1370

33. Tordesillas L, Berin MC. Mechanisms of oral tolerance. Clin Rev Allergy Immunol. 2018;55(2):107-117. doi:10.1007/s12016-018$8680-5$

34. Terada T, Omura S, Kikuoka Y, et al. Sustained effects of intralymphatic pollen specific immunotherapy on Japanese cedar pollinosis. Rhinology. 2020;58(3):241-247. doi:10.4193/Rhin19.301

35. Jones SM, Sicherer SH, Burks AW, et al. Epicutaneous immunotherapy for the treatment of peanut allergy in children and young adults. $J$ Allergy Clin Immunol. 2017;139(4):1242-1252. doi:10.1016/j. jaci.2016.08.017 
36. Prickett SR, Rolland JM, O'Hehir RE. Immunoregulatory T cell epitope peptides: the new frontier in allergy therapy. Clin Exp Allergy. 2015;45(6):1015-1026. doi:10.1111/cea.12554

37. Hirahara K, Saito S, Serizawa N, et al. Oral administration of a dominant T-cell determinant peptide inhibits allergen-specific TH1 and $\mathrm{TH} 2$ cell responses in Cry j 2-primed mice. J Allergy Clin Immunol. 1998;102(6):961-967. doi:10.1016/S0091-6749(98) 70334-3

38. Murasugi T, Nakagami Y, Yoshitomi T, et al. Oral administration of a $\mathrm{T}$ cell epitope inhibits symptoms and reactions of allergic rhinitis in Japanese cedar pollen allergen-sensitized mice. Eur J Pharmacol. 2005;510(1-2):143-148. doi:10.1016/j.ejphar.2005.01.003

39. Takagi H, Hiroi T, Yang L, et al. A rice-based edible vaccine expressing multiple epitopes induces oral tolerance for inhibition of Th2 mediated IgE responses. Proc Natl Acad Sci USA. 2005;102 (26):17525-17530. doi:10.1073/pnas.0503428102

40. Hirahara K, Tatsuta T, Takatori T, et al. Preclinical evaluation of an immunotherapeutic peptide comprising $7 \mathrm{~T}$-cell determinants of Cry $\mathrm{j}$ 1 and Cry j 2, the major Japanese cedar pollen allergens. J Allergy Clin Immunol. 2001;108(1):94-100. doi:10.1067/mai.2001.115481

41. Sone T, Morikubo K, Miyahara M, et al. T cell epitopes in Japanese cedar (Cryptomeria japonica) pollen allergens: choice of major $\mathrm{T}$ cell epitopes in Cry j 1 and Cry j 2 toward design of the peptide-based immunotherapeutics for the management of Japanese cedar pollinosis. J Immunol. 1998;161(1):448-457.

42. Tsunematsu M, Yamaji T, Kozutsumi D, et al. Effect of Cry-consensus peptide, a novel recombinant peptide for immunotherapy of Japanese cedar pollinosis, on an experimental allergic rhinitis model in B10.S mice. Allergol Int. 2007;56(4):465-472. doi:10.2332/allergolint.O-07-495

43. Takagi H, Saito S, Yang L, Nagasaka S, Nishizawa N, Takaiwa F. Oral immunotherapy against a pollen allergy using seed-based peptide vaccine. Plant Biotech J. 2005;3(5):521-533. doi:10.1111/ j.1467-7652.2005.00143.x

44. Takaiwa F, Takagi H, Hirose S, Wakasa Y. Endosperm tissue is good production platform for artificial recombinant proteins in transgenic rice. Plant Biotechnol J. 2007;5(1):84-89. doi:10.1111/j.14677652.2006.00220.x

45. Endo T, Asaka D, Nakayama T, et al. Immunological and symptomatic effects of oral intake of transgenic rice containing 7 linked major T-cell epitopes from Japanese cedar pollen allergens. Int Arch Allergy Immunol. 2021;182(2):109-119. doi:10.1159/000509996

46. Endo T, Asaka D, Nakayama T, et al. Long-term oral administration of transgenic rice containing cedar pollen T-cell epitopes potentially improve medication and allergy related quality of life scores. Allergy Ashma Proc. 2021.

47. Takaiwa F, Yang L. Development of a rice-based peptide vaccine for Japanese cedar and cypress pollen allergies. Transgenic Res. 2014;23 (4):573-584. doi:10.1007/s11248-014-9790-3

48. Wakasa Y, Takagi H, Hirose S, et al. Oral immunotherapy with transgenic rice seed containing destructed Japanese cedar pollen allergens, Cry j 1 and Cry j 2, against Japanese cedar pollinosis. Plant Biotech J. 2013;11(1):66-76. doi:10.1111/pbi.12007

49. Takaiwa F, Yang L, Takagi H, et al. Development of rice-seed-based oral allergy vaccines containing hypoallergenic Japanese cedar pollen allergen derivatives for immunotherapy. J Agric Food Chem. 2019;67 (47):13127-13138. doi:10.1021/acs.jafc.9b05421

50. Takaishi S, Saito S, Kamada M. Evaluation of basophil activation caused by transgenic rice seeds expressing whole $\mathrm{T}$ cell epitopes of the major Japanese cedar pollen allergens. Clin Transl Allergy. 2019;9:11. doi:10.1186/s13601-019-0249-8

51. Fukuda K, Ishida W, Harada Y, et al. Prevention of allergic conjunctivitis in mice by a rice-based edible vaccine containing modified Japanese cedar pollen allergens. Br J Ophthalmol. 2015;99(5):705709. doi:10.1136/bjophthalmol-2014-305842
52. Fukuda K, Ishida W, Harada Y, et al. Efficacy of oral immunotherapy with a rice-based edible vaccine containing hypoallergenic Japanese cedar pollen allergens for treatment of established allergic conjunctivitis in mice. Allergol Int. 2018;67(1):119-123. doi:10.1016/j. alit.2017.06.006

53. Saito S, Takagi H, Wakasa Y, et al. Safety and efficacy of rice seedbased oral allergy vaccine for Japanese cedar pollinosis in Japanese monkeys. Mol Immunol. 2020;125:63-69. doi:10.1016/j. molimm.2020.06.019

54. Toda M, Sato H, Takebe Y, et al. Inhibition of immunoglobulin $\mathrm{E}$ response to Japanese cedar pollen allergen (Cry j 1) in mice by DNA immunization: different outcomes dependent on the plasmid DNA inoculation method. Immunology. 2000;99(2):179-186. doi:10.1046/ j.1365-2567.2000.00935.x

55. Toda M, Kasai M, Hosokawa $\mathrm{H}$, et al. DNA vaccine using invariant chain gene for delivery of CD4+ T cell epitope peptide derived from Japanese cedar pollen allergen inhibits allergen-specific IgE response. Eur J Immunol. 2002;32(6):1631-1639. doi:10.1002/1521-4141 (200206)32:6<1631::AID-IMMU1631 >3.0.CO;2-O

56. Su Y, Connolly M, Marketon A, Heiland T. CryJ-LAMP DNA vaccines for Japanese red cedar allergy induce robust Th1-type immune responses in murine model. $J$ Immunol Res. 2016;2016:4857869. doi:10.1155/2016/4857869

57. Su Y, Romeu-Bonilla E, Anagnostou A, et al. Safety and long-term immunological effects of CryJ2-LAMP plasmid vaccine in Japanese red cedar atopic subjects: a Phase I study. Vaccin Immunother. 2017;13(12):2804-2813. doi:10.1080/21645515.2017.1329070

58. Aoki R, Saito A, Azakami H, Kato A. Effects of various saccharides on the masking of epitope sites and uptake in the gut of cedar allergen Cry $\mathrm{j}$ 1-saccharide conjugates by a naturally occurring Maillard reaction. $J$ Agric Food Chem. 2010;58(13):7986-7990. doi:10.1021/jf100793d

59. Murakami D, Sawatsubashi M, Kikkawa S, et al. Safety and efficacy of a new regimen of short-term oral immunotherapy with Cry $\mathrm{j}$ 1galactomannan conjugate for Japanese cedar pollinosis: a prospective, randomized, open-label study. Allergol Int. 2015;64(2):161-168. doi:10.1016/j.alit.2014.10.009

60. Kaburaki Y, Fujimura T, Kurata K, et al. Induction of Th1 immune responses to Japanese cedar pollen allergen (Cry j 1) in mice immunized with Cry $\mathrm{j} 1$ conjugated with $\mathrm{CpG}$ oligodeoxynucleotide. Comp Immunol Microbiol Infect Dis. 2011;34(2):157-161. doi:10.1016/j. cimid.2010.06.005

61. Suzuki M, Ohta N, Min WP, et al. Immunotherapy with CpG DNA conjugated with T-cell epitope peptide of an allergenic Cry $\mathrm{j} 2$ protein is useful for control of allergic conditions in mice. Int Immunopharmacol. 2007;7(1):46-54. doi:10.1016/j. intimp.2006.08.010

62. Ishii M, Koyama A, Iseki H, et al. Anti-allergic potential of oligomannose-coated liposome-entrapped Cry j 1 as immunotherapy for Japanese cedar pollinosis in mice. Immunopharmacol. 2010;10 (9):1041-1046. doi:10.1016/j.intimp.2010.06.003

63. Fujimura T, Fujinami K, Ishikawa R, et al. Recombinant fusion allergens, Cry j 1 and Cry $\mathrm{j} 2$ from Japanese cedar pollen, conjugated with polyethylene glycol potentiate the attenuation of Cry $\mathrm{j} 1$-specific IgE production in Cry j 1-sensitized mice and Japanese cedar pollen allergen-sensitized monkeys. Int Arch Allergy Immunol. 2015;168 (1):32-43. doi:10.1159/000441141

64. Takagi H, Hiroi T, Yang L, et al. Efficient induction of oral tolerance by fusing cholera toxin B subunit with allergen-specific T-cell epitopes accumulated in rice seed. Vaccine. 2008;26(48):6027-6030. doi:10.1016/j.vaccine.2008.09.019

65. Valenta R, Campana R, Focke-Tejkl M, Niederberger V. Vaccine development for allergen-specific immunotherapy based on recombinant allergens and synthetic allergen peptides: lessons from the past and novel mechanisms of action for the future. J Allergy Clin Immunol. 2016;137(2):351-357. doi:10.1016/j.jaci.2015.12.12990 


\section{Publish your work in this journal}

ImmunoTargets and Therapy is an international, peer-reviewed open access journal focusing on the immunological basis of diseases, potential targets for immune based therapy and treatment protocols employed to improve patient management. Basic immunology and physiology of the immune system in health, and disease will be also covered. In addition, the journal will focus on the impact of management programs and new therapeutic agents and protocols on patient perspectives such as quality of life, adherence and satisfaction. The manuscript management system is completely online and includes a very quick and fair peer-review system, which is all easy to use. Visit http://www.dovepress.com/testimonials.php to read real quotes from published authors. 